\title{
Special Topic: Nanozyme-Based Analysis and Testing
}

\author{
Juewen Liu ${ }^{1}$ \\ Published online: 9 October 2019 \\ (c) The Nonferrous Metals Society of China 2019
}

Developing stable and cost-effective sensors and biosensors is one of the main goals of analytical chemists. Many biosensors rely on enzymes either for recognition of target analytes such as using glucose oxidase for detecting glucose, or for signal generation such as using horse radish peroxidase (HRP) in immunoassays. While these protein-based enzymes are highly efficient, they are susceptible to denaturation and some enzymes are quite costly.

Using nanomaterials to mimic enzyme activities has attracted growing interest over the last 10 years or so. Many common metal oxides, metals, and carbon-based nanomaterials have oxidase, peroxidase and catalase like activities, and nanozyme was coined for describing them. The main difference between nanozymes and typical inorganic catalysts is the former focuses on biologically relevant reactions taking place at close to physiological conditions. Nanozymes, in general, have higher stability and lower cost compared to protein-based enzymes. For the analytical community, nanozymes have been used for both target recognition and for signal transduction. We are seeing more and more examples of nanozymes used in environmental monitoring, biomedical diagnosis and food safety applications.

Journal of Analysis and Testing provides an international academic platform for the publication of original research papers, rapid communications, and critical reviews in all aspects of fundamental and applied analytical chemistry. To highlight the current developments and stimulate new thoughts of the nanozyme field, this Special Issue is focused on biologically and environmentally related nanozymebased analysis and testing. This Special Issue features two review papers and eight research articles.

Professor Chang from National Taiwan University reviewed carbon dot (Cdot) based peroxidase mimics for analytical applications. Carbon dots are often produced by

Juewen Liu

liujw@uwaterloo.ca

1 Department of Chemistry, University of Waterloo, Waterloo, ON N2L 3G1, Canada simple hydrothermal methods from cost-effective starting materials with interesting fluorescence and sometimes also phosphorescence properties. The importance of the oxidation state and surface residues of Cdots are highlighted, and their nanocomposites with metals, metal oxides, and metal sulfides are described. Examples of selective absorption, fluorescence, and electrochemical sensing systems are presented to show their potential analytical applications. Huang and coworkers from National Taiwan Ocean University reviewed nanozyme-based sensors for detection of heavy metal ions. Heavy metals are important analytes in the environment due to their bioaccumulative and non-degradable properties, posing adverse health effects. This paper articulates the importance of the surface properties of nanozymes, and metal binding can either promote or inhibit the activity of nanozymes, which is the basis of sensing.

For the research papers, a few of them focused on the development of new nanozymes. Lopez and Liu from the University of Waterloo compared adenine and 2-aminopurine for forming coordination nanoparticles with various metal ions and $\mathrm{Au}^{3+}$ was found to form similar nanoparticles with both ligands. 2-Aminopurine is a fluorescent analog of adenine and its fluorescence allowed convenient monitoring of the formation and dissolution of the product. The products showed oxidase-like activity for oxidation of a chromogenic substrate. Alkaline phosphatase (ALP) is an important clinical biomarker for many diseases. Taking advantage of the strong oxidase-like activity of $\mathrm{MnFe}_{2} \mathrm{O}_{4}$, and its inhibition by pyrophosphate (PPi), Niu and coworkers from Jiangsu University developed a colorimetric sensor for detecting ALP. ALP can hydrolyze PPi and recover the activity of $\mathrm{MnFe}_{2} \mathrm{O}_{4}$ for signal-on detection of ALP down to $0.27 \mathrm{u} / \mathrm{L}$.

Most nanozymes collected in this Special Issue have peroxidase-like activity, where oxidation takes place in the presence of $\mathrm{H}_{2} \mathrm{O}_{2}$. Professor Yigit's team from University at Albany, State University of New York studied the peroxidase-like activity of zirconium(IV) oxide nanoparticles. The effects of $\mathrm{pH}$, temperature and $\mathrm{H}_{2} \mathrm{O}_{2}$ concentration have been systematically studied. This nanozyme was tested in different protein solutions and showed robust activity. 
This stable material is likely to find interesting analytical applications. Wei and coworkers from Nanjing University prepared ruthenium selenide nanomaterials with ordered mesoporous structure using the KIT-6 silica as a template. The product showed excellent peroxidase-like activity. Based on this, colorimetric detection of $\mathrm{H}_{2} \mathrm{O}_{2}$ and glucose was demonstrated. A collaborative work between Qiu from Nanchang University and Liu from the University of Waterloo described coordination nanomaterials formed by mixing $\mathrm{Cu}^{2+}$ with excessed nucleotides and they found that guanosine 5'-monophosphate (GMP) to be most active for its oxidation nanozyme activity. A sensitive and selective strategy for $\mathrm{Cu}^{2+}$ sensing was proposed. This robust sensor allowed detection even in seawater with a very high concentration of salt. Jiang and coworkers from Southeast University synthesized adenosine phosphate (AXP) templated gold nanoparticles (AuNPs) with small size, good optical properties, and excellent peroxidase-like activities. They found that adding divalent metal ions into the AuNPs allowed regeneration of the activity that was lost during the reaction. In addition, it was found that glutathione (GSH) can suppress the activity, allowing its detection. Fu and coworkers from Tianjin University employed $N$-acety-L-cysteine (NAC) as a ligand to synthesize and stabilize a series of peroxidase-like Pt nanoclusters. The cluster size and the charge state of the product were optimized and they found that the ratio between the Pt salt and NAC ligand was important. Since heparin can activate the nanozyme at $\mathrm{pH} 6.0$, an ultrasensitive assay was developed for detecting heparin, with a detection limit of $2 \mathrm{ng} / \mathrm{mL}$. This proposed method was also applicable in biological fluids. Zhao and coworkers from Dalian Institute of Technology synthesized iron-based metal-organic frameworks (MOFs) with acidized carbon nanotubes (CNTs) via a simple hydrothermal process. The CNTs can enhance the affinity between the MOF and DNA, allowing DNA to regulate the peroxidase-like nanozyme activity of the MOF. The nanozyme achieved ultrasensitive detection of $\mathrm{H}_{2} \mathrm{O}_{2}$ with the LOD of $17.64 \mu \mathrm{g} / \mathrm{L}$.

This Special Issue only represents a small collection of work in the field of nanozyme-based sensors. We hope that they can be useful for readers to get an idea of the current state of research in this field. By reading these papers, some challenges and future research opportunities can also be defined. For example, developing new chemistry for interfacing nanozymes with analytical systems is needed. Simple platforms such as paper-based sensors are desirable to match the advantages of nanozymes. Since the nanozyme reaction takes place on the surface of nanomaterials, adsorption of other molecules may affect the catalytic activity, leading to problems in reproducibility. For building more sophisticated sensors, bioconjugation on nanozymes is needed. Finally, there is still room for improving the activity and specificity of nanozymes.

I am very grateful to the authors for contributing highquality papers to this Special Issue, and the reviewers for their efforts and help in reviewing these manuscripts. I also wish to thank the Editorial Office for their help along the way.

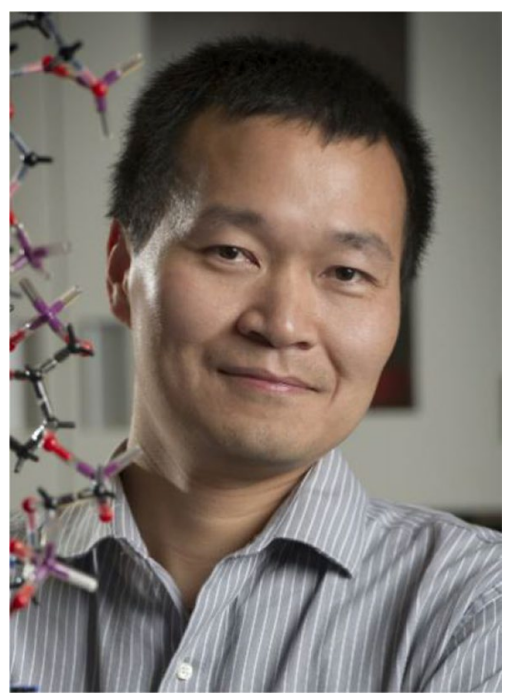

Juewen Liu received his $\mathrm{Ph} . \mathrm{D}$. degree from the University of Illinois at Urbana-Champaign in 2005. After postdoctoral research at Sandia National Labs and the University of New Mexico, he joined the Department of Chemistry at the University of Waterloo in 2009. He is currently a full professor there. He received a Fred Beamish Award (2014) and a W.A.E. McBryde Medal (2018) from the Canadian Society for Chemistry for his contribution in bioanalytical chemistry, and an Ontario Early Researcher Award in 2011. He serves an Editor for Biosensors \& Bioelectronics, Trends in Analytical Chemistry (TrAC), an Associate Editor for Chinese Chemical Letters, Journal of Analysis and Testing, and is on the Editorial Advisory Board of Langmuir and Particles \& Particle Systems Characterization. He is interested in metal-dependent DNAzymes, biointerface science, and nanozymes. Since 2002, he has published over 300 papers, receiving over 20,000 citations with an $\mathrm{H}$-index of 66 . 